\title{
El uso de opioides en los adultos mayores. Un tema a tener en cuenta
}

\author{
I. Failde \\ Catedrática Medicina Preventiva y Salud Pública. Universidad de Cádiz
}

Los opioides son fármacos considerados esenciales para el control del dolor de moderado a intenso, especialmente en pacientes con dolor crónico oncológico. A pesar de sus ventajas, en los últimos años hemos asistido a una alerta sanitaria esencialmente en EE. UU., motivada por las elevadas cifras de uso indebido, abuso, adicción y mortalidad relacionadas con la prescripción y el consumo de estos fármacos. En España, la prescripción y uso de opioides ha aumentado entre 2008 y 2015 (1), aunque las cifras no alcanzan los niveles de otros países de Europa o EE. UU. (2).

El dolor crónico es muy prevalente en la población de adultos mayores; sin embargo, su tratamiento adecuado es un reto en esta población altamente vulnerable. El tratamiento del dolor con opioides en pacientes mayores ha sido objeto de debate desde hace algunos años, a pesar de que en EE. UU. se ha mostrado que el $25 \%$ de los consumidores de opioides de larga duración eran mayores de 65 años, y su uso prolongado se ha asociado con hospitalizaciones por sobredosis [3]. Asimismo, la relación riesgo-beneficio de estos fármacos ha sido cuestionada en estos pacientes debido a la frecuente comorbilidad que sufren, su habitual polifarmacia y a los mayores potenciales efectos adversos de estos fármacos. Sin embargo, son muy escasos los estudios que analizan el uso prolongado de opioides en este grupo de pacientes y los riesgos asociados a su utilización.

Oh GY y cols., en este estudio, investigan patrones de uso de opioides en mayores de 65 años, y analizan los factores que predicen la trayectoria de consumo a lo largo de 10 años de seguimiento. Los autores encuentran cuatro patrones según la trayectoria del consumo: mínimo, discontinuo, incidente-crónico y prevalente crónico, marcando la diferencia entre estos dos últimos el hecho de que mientras los primeros inician el consumo más o menos al cuarto año del estudio para luego continuarlo, en los segundos el uso es crónico y desde el inicio del estudio. Los autores observan estos mismos patrones en los consumidores de cualquier tipo de analgésicos opioide, en los que la prevalencia de consumo es del 3,8 $\%$, y en los usuarios de opioides potentes, en los que la prevalencia es del 2,4\%. Entre los factores predictores de uso crónico de opioides potentes, el sexo femenino, la mayor edad, la raza negra, vivir en comunidad o en centros de cuidados, eran los más importantes. De igual manera, el consumo de antidepresivos, ansiolíticos o hipnóticos también fueron predictores de uso crónico de opioides de cualquier tipo, incluidos los opioides potentes. Los autores, basándose en estudios previos (4) en los que se muestra que el uso previo de benzodiacepinas es un factor de riesgo de uso prolongado de opioides, llaman la atención sobre la necesidad de evaluar los potenciales riesgos de esta asociación en adultos. Asimismo, y a pesar de que sus resultados son consistentes con otros estudios, cuestionan el mayor riesgo de uso prologando de estos fármacos con la polifarmacia, atribuyendo esta circunstancia al seguimiento más estricto que con frecuencia se realiza en los pacientes polimedicados y con más comorbilidades.

Los autores de este trabajo, como conclusión, resaltan el desproporcionado uso de opioides en los participantes más vulnerables (por ejemplo mujeres, ancianos, con múltiples comorbilidades y polifarmacia), la necesidad de realizar estudios en este colectivo y de desarrollar guías basadas en la evidencia para el uso de opioides en pacientes mayores.

[Oh G, Abner EL, Fardo DW, Freeman PR, Moga DC. Patterns and predictors of chronic opioid use in older adults: A retrospective cohort study. PLoS One. 2019;14[1]:e0210341. DOI: 10.1371/journal.pone.0210341] 
La atención del dolor en los pacientes de más edad debe ser una prioridad en la atención sanitaria, y la evaluación de las ventajas y los riesgos asociados al tratamiento con opioides debe ser un tema a considerar.

\section{BIBLIOGRAFÍA}

1. Utilización de medicamentos opioides en España durante el periodo 2008-2015. Agencia Española de Medicamentos y Productos Sanitarios. Madrid: Ministerio de Sanidad, Asuntos Sociales e Igualdad; 2017.
2. Birke H, Kurita GP, Sjøgren P, Højsted J, Simonsen MK, Juel $\mathrm{K}$, et al. Chronic non-cancer pain and the epidemic prescription of opioids in the Danish population: trends from 2000 to 2013. Acta Anaesthesiol Scand 2016;60(5):623e633. DOI: 10.1111/aas.12700.

3. Steinman MA, Komaiko KD, Fung KZ, Ritchie CS. Use of opioids and other analgesics by older adults in the United States, 1999-2010. Pain Med. 2015;16(2):319-27. DOl: 10.1111/ pme.12613.

4. Lalic S, Gisev N, Bell JS, Korhonen MJ, llomaki J. Predictors of persistent prescription opioid analgesic use among people without cancer in Australia. $\mathrm{Br} J$ Clin Pharmacol. 2018;84(6):1267-78. DOl: org/10.1111/bcp.13556. 